\title{
Degeneracy and consistency condition for Berry phases: Gap closing under a local gauge twist
}

\author{
T. Hirano, ${ }^{1, *}$ H. Katsura, ${ }^{1, \dagger}$ and Y. Hatsugai ${ }^{2,+}$ \\ ${ }^{1}$ Department of Applied Physics, The University of Tokyo, 7-3-1 Hongo, Bunkyo-ku, Tokyo 113-8656, Japan \\ ${ }^{2}$ Institute of Physics, University of Tsukuba, 1-1-1 Tennodai, Tsukuba, Ibaraki 305-8571, Japan \\ (Received 2 July 2008; revised manuscript received 15 July 2008; published 21 August 2008)
}

\begin{abstract}
We have discussed a consistency condition of Berry phases defined by a local gauge twist and spatial symmetries of the many-body system. It imposes a nontrivial gap closing condition under the gauge twist in both finite- and infinite-size systems. It also implies a necessary condition for the gapped and unique ground state. As for the simplest case, it predicts an inevitable gap closing in the Heisenberg chain of half-integer spins. Its relation to the Lieb-Schultz-Mattis theorem is discussed based on the symmetries of the twisted Hamiltonian. The discussion is also extended to the (approximately) degenerate multiplet and fermion cases. It restricts the number of the states in the low energy cluster of the spectrum by the filling of the fermions. Constraints by the reflection symmetry are also discussed.
\end{abstract}

DOI: 10.1103/PhysRevB.78.054431

PACS number(s): 75.10.Jm, 64.70.Tg, 03.65.Vf, 71.10.Fd

\section{INTRODUCTION}

For the last decade, quantum criticality with gapless excitations have been focused in its relation to a quantum phase transition. Generically speaking, the gapless phase is only realized by a fine tuning of the parameters of the quantum Hamiltonian. Some machinery to protect the gap closing of the quantum-mechanical system is necessary. Symmetries as a spatial translation can be one of the reasons which plays an important role in the Fermi liquids. Today we have other machineries for the gap closing in a generic situation. One is the Nambu-Goldstone mechanism. When a continuous symmetry is broken spontaneously, there can be a gapless excitation by slowly varying its local order parameter which is responsible for the symmetry breaking. It is closely related to the Lieb-Schultz-Mattis (LSM) argument ${ }^{1-7}$ to prove existence of the gapless excitations in a half-odd-integer spin chains. This LSM argument is justified in restricted situations. It allows existence of a gapped phase which is known as the Haldane phase in the integer-spin chains. This is a typical quantum liquid where strong quantum fluctuations prevent from forming an ordered phase. The other mechanism to protect the gapless excitations is an appearance of the edge states such as the one in quantum Hall states and Haldane phases. ${ }^{8-16}$ There is a fundamental class of physical phases with intrinsic energy gap as topological insulators. They are gapped quantum liquids in a bulk without any fundamental symmetry breaking. However the system has a geometrical (topological) perturbation such as the existence of the boundaries; low energy modes (quasiparticle) as the generic edge states appear and the phase becomes gapless when the boundaries are infinitely separated.

The existence of the energy gap is also closely related to the degeneracy of the ground state. When the discrete symmetry is broken such as for the Heisenberg spins with the Ising anisotropy or charge ordered states, the symmetry broken states split into a low energy cluster with small energy splitting in a finite periodic system. The exact degeneracy is only realized in the infinite-size limit. For the topological insulators without any symmetry breaking, there can be additional degeneracy as the topological degeneracy which characterizes the nontrivial quantum liquids. ${ }^{17,18}$

As for the topological insulators, quantum order parameters by the many-body Berry phases, associated with the local gauge twists, have been proposed and its validity is justified for several concrete models. ${ }^{19-24}$ Using this Berry phase and its symmetry property, we prove that there is an inevitable degeneracy during the twist as for some classes of Hamiltonian which is also related to the LSM theorem. Our discussions are valid not only in infinite-size systems but also in finite-size systems, which can be applicable for various correlated electron systems.

\section{LOCAL ORDER PARAMETER DEFINED BY THE BERRY PHASE}

\section{A. Definition}

Let us define a local order parameter $\gamma_{i j}$ at a link $(i j)$ by the Berry phase ${ }^{19-22,25}$

$$
i \gamma_{i j}=\int_{0}^{2 \pi} d \phi A_{i j}(\phi),
$$

where $A_{i j}(\phi)$ is a Berry connection obtained from a gauge fixed (single valued) normalized ground state $\left|\psi_{i j}\right\rangle$ of a Hamiltonian $H_{i j}(\phi)$ as $A_{i j}(\phi)=\left\langle\psi_{i j}\left|\partial_{\phi}\right| \psi_{i j}\right\rangle,{ }^{19-22}$ where $\phi$ is a parameter of the local $U(1)$ twist on the link. It is gauge dependent but is well defined up to modulo $2 \pi$. Further, it is quantized if the ground state is invariant under some antiunitary operation (see Appendixes A and B).

Let us now consider the Berry phase in quantum spin systems. As for the Heisenberg model with generic connectivity, the Hamiltonian with the local twist is given as

$$
H_{i j}(\phi)=J_{i j}\left[\left(e^{i \phi} S_{i}^{+} S_{j}^{-}+e^{-i \phi} S_{i}^{-} S_{j}^{+}\right) / 2+S_{i}^{z} S_{j}^{z}\right]+\sum_{(k l) \neq(i j)} J_{k l} \boldsymbol{S}_{k} \cdot \boldsymbol{S}_{l},
$$

where $J_{i j}$ is the exchange coupling between $\boldsymbol{S}_{i}$ and $\boldsymbol{S}_{j}$ and is assumed to be nonzero. Physically, this twist can be regarded as an introduction of the Dzyaloshinskii-Moriya interaction and the XXZ anisotropy since the first term can be written in a decoupled form as 


$$
J_{i j}\left[\cos \phi\left(S_{i}^{x} S_{j}^{x}+S_{i}^{y} S_{j}^{y}\right)+S_{i}^{z} S_{j}^{z}\right]+J_{i j} \sin \phi\left(S_{i}^{x} S_{j}^{y}-S_{i}^{y} S_{j}^{x}\right)
$$

We should note that the Berry phase is only well defined unless the energy gap between the ground state and the firstexcited states does not vanish during the twist.

\section{B. Local gauge transformation and constraint on Berry} phases: One-dimensional Heisenberg model as an example

Since the twist on the link can be modified by the local gauge transformation, it gives some constraints for the Berry phase distribution. For simplicity, let us first discuss the onedimensional nearest-neighbor antiferromagnetic Heisenberg model of generic spins on a finite lattice. We discuss within the subspace of fixed total $S^{z}, S_{\mathrm{tot}}^{z}=\Sigma S_{j}^{z}$, since it commutes with $H_{i, j}(\phi)$. Performing a local gauge transformation at the site $j$ as

$$
U_{j}(\phi)=e^{i\left(S-S_{j}^{z}\right) \phi}, \quad \phi \in[0,2 \pi),
$$

which is single valued in the parameter space, one has an important relation between the two different Hamiltonians (which are gauge equivalent), $H_{j, j+1}$ and $H_{j-1, j}$ as $H_{j, j+1}$ $=U_{j}^{\dagger} H_{j-1, j} U_{j}$. Correspondingly, the states are mapped into each other as $\left|\psi_{j, j+1}\right\rangle=U_{j}^{\dagger}\left|\psi_{j-1, j}\right\rangle$, where $\left|\psi_{j-1, j}\right\rangle$ and $\left|\psi_{j, j+1}\right\rangle$ are two different ground states of $H_{j-1, j}$ and $H_{j, j+1}$, respectively. Note that if the state $\left|\psi_{j-1, j}\right\rangle$ is gauge fixed as single valued in the parameter space, it is also true for the state $\left|\psi_{j, j+1}\right\rangle$. Assuming that the ground state is unique and gapped during the twisting, this simple relation gives a constraint for the quantum local order parameters $\gamma_{i j}$ 's:

$$
\begin{aligned}
\gamma_{j, j+1} & =-i \int\left\langle\psi_{j-1, j}\right| U_{j} \partial_{\phi}\left(U_{j}^{\dagger}\left|\psi_{j-1, j}\right\rangle\right) d \phi, \\
& =\gamma_{j-1, j}+\int\left(S-\left\langle\psi\left|S_{j}^{z}\right| \psi\right\rangle\right) d \phi .
\end{aligned}
$$

Since the expectation value of the hermitian operator $S_{j}^{z}$ is independent of the gauge transformation, we have dropped the label of the wave function to specify the position of the twist. The time-reversal invariance of the state $|\psi\rangle$ implies $\left\langle\psi\left|S_{i}^{z}\right| \psi\right\rangle=-\left(\left\langle\psi\left|S_{i}^{z}\right| \psi\right\rangle\right)^{*}=0$. Then, one obtains the relation between $\gamma_{j-1, j}$ and $\gamma_{j, j+1}$ as

$$
\gamma_{j-1, j}=2 \pi S+\gamma_{j, j+1} .
$$

Since the present one-dimensional (1D) Heisenberg model has a translational symmetry, the Berry phases as the quantum order parameters should also respect this translational symmetry, that is, $\gamma_{i j}$ should be independent of the link $(i j)$ in mod $2 \pi$ unless they are well defined. However, this is impossible for the half-integer spins due to the above constraint $\gamma_{j-1, j} \equiv \gamma_{j, j+1}+\pi(\bmod 2 \pi)$. It implies that there is a gap closing under the local twist, as for the antiferromagnetic Heisenberg chains with half-integer spins on a finite lattice. Although our results can be applicable for arbitrary halfinteger spins, similar conclusions for the $S=1 / 2$ case are also obtained from different analysis. ${ }^{7,26,27}$ As for the integer-spin Heisenberg model, the above constraint does not forbid uni-

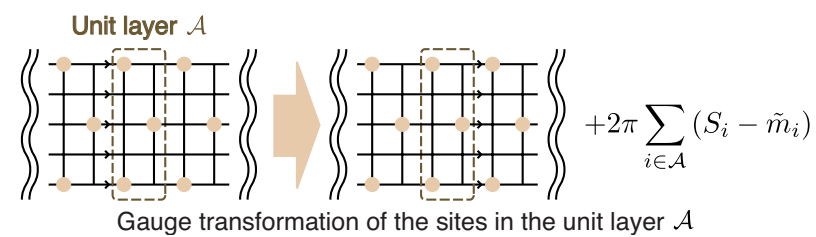

FIG. 1. (Color online) The definition of the unit layer and the gauge transformation of the Berry phase. The arrows on links denotes the link twist.

form distribution of the Berry phase $\gamma_{i j}$. Actually, the uniform $\pi$ Berry phases are realized in $S=1$ cases. ${ }^{19-22,24,28}$

\section{SYMMETRY AND CONSISTENCY CONDITION OF BERRY PHASES}

As we have shown above, symmetry in the system such as the translational symmetry gives an important restriction to the arrangement of Berry phases. The idea above is extended to the general cases without any difficulty. Let us consider sets of some generic twists for several links labeled by "In." Assuming the uniqueness of the ground state even under the twisting, one can define the Berry phase $\gamma_{\text {In }}$. Next let us perform a set of the local gauge transformation within the area $\mathcal{A}$. Then we have a new set of twists labeled by "Out." Under this generic setup, one has a constraint between the two corresponding Berry phases $\gamma_{\mathrm{In}}$ and $\gamma_{\mathrm{Out}}$ as

$$
\gamma_{\text {In }}=\gamma_{\text {Out }}+2 \pi \sum_{i \in \mathcal{A}}\left(S_{i}-\widetilde{m}_{i}\right), \tilde{m}_{i}=\frac{1}{2 \pi} \int\left\langle\psi\left|S_{i}^{z}\right| \psi\right\rangle d \phi,
$$

where $\tilde{m}_{i}$ is a magnetization of the $i$ th site and is nonzero in general.

\section{A. Translational symmetry in quantum spin systems}

Let us now consider a spin ladder as an example of translational invariant system; we take $\mathcal{A}$ as a unit layer and define Berry phase by twisting the links on the left boundary of the area $\mathcal{A}$ simultaneously (see Fig. 1 ). Due to the translational symmetry, we have a constraint for the Berry phases as $\gamma_{\mathrm{In}} \equiv \gamma_{\text {Out }}(\bmod 2 \pi)$ assuming that the ground state is unique and gapped under the twisting. Since the total $S^{z}$ is conserved, we have $\sum_{i \in \mathcal{A}} \widetilde{m}_{i}=(1 / 2 \pi) \int d \phi\left\langle\psi\left|\sum_{i} S_{i}^{z}\right| \psi\right\rangle / N$ $=\left\langle\psi\left|\sum_{i} S_{i}^{z}\right| \psi\right\rangle / N=|\mathcal{A}| m$, where $|\mathcal{A}|$ is a number of sites in the unit layer and $m$ is the average magnetization ( $N$ is a number of the unit layers). Note that the translational invariance of the magnetization at arbitrary $\phi$ is guaranteed by the fact that $S_{\text {tot }}^{z}$ commutes with a unitary transformation which spreads the flux in a transitionally invariant way. Then we conclude that if the ground state is unique and gapped under the twist, the following condition holds:

$$
\sum_{i \in \mathcal{A}}\left(S_{i}-m\right) \equiv \nu \in \mathbb{Z}
$$

It is a condition to have a magnetization plateau. ${ }^{29}$

By considering a non-Abelian Berry phase, ${ }^{19-22}$ this argument can be extended to the case with (approximate) degeneracy. A precise definition of the non-Abelian Berry phase 
and a numerically efficient way to evaluate its value are given in Appendixes $\mathrm{A}$ and $\mathrm{C}$, respectively. Now let us assume the low energy spectrum near the ground states forms a multiplet $\Psi=\left(\left|\psi_{1}\right\rangle, \cdots,\left|\psi_{M}\right\rangle\right)$ with $M$ eigenstates, $\left|\psi_{i}\right\rangle(i=1, \cdots, M)$. Here, we assume that they are in the subspace of the same $S_{\text {tot }}^{z}$ and the energy gap above the multiplet is stable. Then as for the Berry phase $\gamma=\int d \phi \operatorname{Tr} \Psi^{\dagger} \partial_{\phi} \Psi$, we have a relation

$$
\gamma_{\text {In }}=\gamma_{\text {Out }}+2 \pi M \nu
$$

based on the same assumption. Therefore as for the translational invariant system, we have a consistency requirement $2 \pi M \nu \equiv 0(\bmod 2 \pi)$ assuming the gap even under the twist. Then as for $\nu=\sum_{i \in \mathcal{A}}\left(S_{i}-m\right)=p / q$ with relatively prime $p$ and $q, M$ has to be a multiple of $q$, that is, the low energy spectrum has to form a cluster of $q \ell$ states $(\ell$ : integer). This situation naturally occurs with discrete symmetry breaking ${ }^{30}$ or topological degeneracy.

As for the $S=1 / 2$ Heisenberg ladder with $n$ legs, the discussion here predicts a gap closing under the twist when $n$ is odd. ${ }^{31,32}$ It allows to have an energy gap above the low energy multiplet composed of two states even with the $S$ $=1 / 2$ system, which is realized for the spin tube. ${ }^{33}$ The present argument also gives a consistent description for ferrimagnets discussed by the effective-field theory and the LSM argument. ${ }^{34}$ Consider a Heisenberg spin chain with different spin quantum numbers as $S_{j}=S_{1}$ for $j=1(\bmod M)$ and $S_{j}=S_{2}$ for others. Taking the unit layer to include these $M$ spins, the gauge transformation yields the relation of the Berry phase as $\gamma_{1}=\gamma_{2}+2 \pi\left[S_{1}+(M-1) S_{2}\right]$. The system must have a gap closing under the twist if $\left[S_{1}+(M-1) S_{2}\right] \notin \mathbb{Z}$. On the other hand, if the ground state of the system is unique and gapped under the twist then the condition $\left[S_{1}+(M-1) S_{2}\right] \in \mathbb{Z}$ holds.

\section{B. LSM theorem and degeneracy at $\phi=\pi$}

We shall now discuss the connection to the LSM theorem. ${ }^{1}$ Our argument suggests that there is at least one level-crossing point during the local twist if Berry phases cannot be arranged in a compatible way with the translational symmetry. Indeed, one can rigorously show the degeneracy of the ground state at $\phi=\pi$. For simplicity, we consider the first example, namely, the half-integer Heisenberg spin chain with length $L$ in a zero magnetic field. We introduce the following two symmetry operations: One is $U_{j}(\phi) T$, where $T$ is the operation for the one-step translation along the chain. The Hamiltonian $H_{j-1, j}$ is invariant under this operation:

$$
\left[U_{j}(\phi) T\right]^{\dagger} H_{j-1, j}(\phi)\left[U_{j}(\phi) T\right]=H_{j-1, j}(\phi) .
$$

The other is the spin-flip operation $F$ defined by $F S_{j}^{z} F=-S_{j}^{z}$ and $F S_{j}^{ \pm} F=S_{j}^{\mp}$ for any $j$. The Hamiltonian has this symmetry if and only if $\phi=0$ or $\pi$, i.e.,

$$
F H_{j-1, j}(0) F=H_{j-1, j}, \quad F H_{j-1, j}(\pi) F=H_{j-1, j} .
$$

At $\phi=\pi$, there is a hidden algebraic relation $\left\{U_{j}(\pi) T, F\right\}$ $=0$ where $\{$,$\} denotes the anticommutator. This can be shown$ by the fact that $F U_{j}(\pi) F=U_{j}(\pi) e^{2 \pi i S_{j}^{z}}=-U_{j}(\pi)$ since we consider the half-odd-integer spin chains. From the anticommutation relation, there exist at least two ground states labeled by the quantum number associated to $F$ at $\phi=\pi$. We call two of them $|G(\pi,+1)\rangle$ and $|G(\pi,-1)\rangle$ $\equiv\left[U_{j}(\pi) T\right]|G(\pi,+1)\rangle$, where $F|G(\pi, \eta)\rangle=\eta|G(\pi, \eta)\rangle$. As is obvious from the above argument, this degeneracy is not restricted to the ground state. Actually every energy level is at least doubly degenerate at $\phi=\pi$ and can be distinguished by the eigenvalue of $F$. An extension to the above argument to other systems with translational symmetry can be done in a straightforward way by replacing $U_{j}(\phi) T$ with $U_{\mathcal{A}}(\phi) T_{\mathcal{A}}$, where $U_{\mathcal{A}}(\phi)=\prod_{j \in \mathcal{A}} U_{j}(\phi)$ and $T_{\mathcal{A}}$ is the translation of the unit layer.

To discuss the relation between this degeneracy and the LSM theorem, it is useful to introduce the translationally invariant Hamiltonian with the twist $\phi$ as $\tilde{H}(\phi)$ $\equiv U^{\dagger}(\phi) H_{L, 1}(\phi) U(\phi)$, where $U(\phi)=\Pi_{j=1}^{L} U_{j}(-j \phi / L)$. The level crossing at $\phi=\pi$ suggests that one of the excited state of $\tilde{H}(0)=H_{L, 1}(0)$ is smoothly connected to the ground state of $\tilde{H}(2 \pi)$. Since $H_{L, 1}(2 \pi)=H_{L, 1}(0)$, the ground state of $\tilde{H}(2 \pi)$ is given by $U^{\dagger}(2 \pi)|G(0)\rangle$, where $|G(0)\rangle$ is the ground state of $\widetilde{H}(0)$. Finally, along the same lines as the LSM argument, ${ }^{1}$ one can show that $U^{\dagger}(2 \pi)|G(0)\rangle$ is orthogonal to $|G(0)\rangle$ and the energy difference between them is $\mathcal{O}(1 / L)$ using the translational and $F$ symmetry.

Another comment is that, in the case of one-dimensional spin chains with open boundary condition, the local gauge twists are always gauged away. Using the gauge transformation of the string type $\Pi_{i=1}^{j} U_{i}(\phi)=\exp \left[i \sum_{i=1}^{j}\left(S-S_{i}^{z}\right) \phi\right]$ where the product is taken from the boundary site to the twisted link, we obtain the Berry phase as $\gamma_{j, j+1}=2 \sum_{i=1}^{j} S_{i} \pi$ assuming the energy gap. It is consistent with the generic valencebond-solid (VBS) state. ${ }^{24}$ As for the $S=1$ spin chain with open boundaries, the Haldane gap corresponds to the energy gap above the Kennedy triplet. ${ }^{13}$ Then the Berry phase of the low energy cluster below the Haldane gap, which includes contribution of the edge states, gives vanishing Berry phase. It should be distinguished from the translationally invariant case without edge. ${ }^{19-22,28}$

\section{Translational symmetry in fermionic systems}

Further, this present argument is also applicable for systems with charge degrees of freedom. For simplicity, we shall consider a spinless fermion model. However, it is straightforward to extend our argument to the cases where fermions have spin degrees of freedom and even where they interact with localized spins such as the Kondo-lattice model. ${ }^{5,35}$ Let us consider a model with conserved particle number $n_{\text {tot }}=\sum_{i} n_{i}$ such as spinless fermions with interaction $H=\sum_{\langle i j\rangle}\left(t c_{i}^{\dagger} c_{j}+\right.$ H.c. $\left.+V_{i j} n_{i} n_{j}\right)$, where $n_{i}=c_{i}^{\dagger} c_{i}$ and $c_{i}$ is a fermion annihilation operator at site $i$. The $U(1)$ gauge twist against the charge degree of freedom is introduced by replacing a hopping at the special link $(i j)$ as $e^{i \phi} c_{i}^{\dagger} c_{j}$ and the $U(1)$ local gauge transformation is given by

$$
U_{j}(\phi)=e^{i n_{j} \phi} .
$$

Then the transformation property of the Berry phase under the gauge transformation leads the relation 


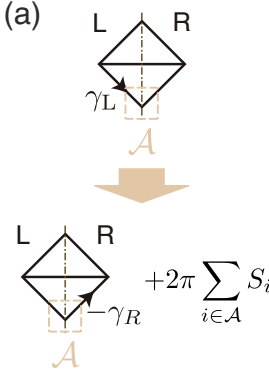

Gauge transformation of the sites in $\mathcal{A}$

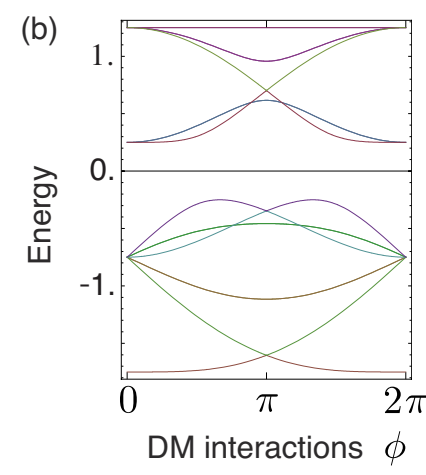

FIG. 2. (Color online) (a) The definition of $\mathcal{A}$ in the reflection symmetric system and the gauge transformation of the Berry phase. The system is reflection symmetric about a dashed line. (b) Spectral flow of a reflection symmetric $S=1 / 2$ Heisenberg model with DM interactions $\phi$. The shape of lattice is the one in (a). At $\phi=\pi$, all states are at least doubly degenerate. Some of them are doubly degenerate for all of the twist $\phi$.

$$
\gamma_{\text {In }}=\gamma_{\text {Out }}-2 \pi \sum_{i \in \mathcal{A}} \tilde{n}_{i}
$$

where $\tilde{n}_{i}=\frac{1}{2 \pi} \int\left\langle\phi\left|n_{i}\right| \phi\right\rangle d \phi$ as the case of spins and $\mathcal{A}$ is a unit layer. Following the same argument as the spin case, the translational symmetry gives a necessary requirement for the unique and gapped ground state under the twist as

$$
\rho \in \mathbb{Z}, \quad \rho=\frac{1}{N}\left\langle\sum_{j} n_{j}\right\rangle,
$$

where $\rho$ is an average particle number per unit layer and $N$ is a number of the unit layers. It has also a non-Abelian extension for the degenerate multiplet which is just a repetition of the spin case. It requires that, when the filling is $\rho=p / q$ with relatively prime $p$ and $q$, there exists a multiplet of $M$ states in the low energy spectrum to form a cluster, which is separated from the else under the twist as $M=q \ell, \ell=1,2, \cdots$, and $\rho=p / q .^{30,36-38}$

\section{Reflection symmetry}

We may further apply the present argument for the generic symmetry such as reflection symmetry. Consistency between the possible Berry phases and the reflection symmetry of the physical system requires some restriction. Let us consider a reflection symmetric system consisting of two subsystems $R$ and $L$ which are mirror images of one another. We first choose a set of sites $\mathcal{A}$ where we perform a gauge transformation. $\mathcal{A}$ itself is chosen to be reflection symmetric. See Fig. 2(a) for the simplest example. Then we define the Berry phase $\gamma_{L}$ by twisting some links on the boundary of $\mathcal{A}: \partial \mathcal{A}$. We denote this Berry phase as $\gamma_{L}$ and the symmetric partner of $\gamma_{L}$ as $\gamma_{R}$. Our gauge transformation of the Berry phase results in

$$
\gamma_{L}=-\gamma_{R}+2 \pi \sum_{j \in \mathcal{A}}\left(S_{j}-\tilde{m}_{j}\right) .
$$

The relations $-\gamma_{R} \equiv \gamma_{R}, \bmod 2 \pi$, and $\tilde{m}_{j}=0$ hold if the timereversal symmetry is also present as shown in Appendix B.
In such case, we obtain that $\gamma_{L}=\gamma_{R}+2 \pi \Sigma_{\mathcal{A}} S_{j}$ if the ground state is unique and gapped under the twist. Since the reflection symmetry of the physical structure implies $\gamma_{L} \equiv \gamma_{R}$ $(\bmod 2 \pi)$, in the following case:

$$
\sum_{j \in \mathcal{A}} S_{j} \notin \mathbb{Z},
$$

we predict a level crossing during the twisting. Our argument can be extended without any difficulty for generic reflection symmetric models including even three-dimensional ones. Numerical results showing the pattern of level crossings are given in Fig. 2(b). Note that there is the ground-state degeneracy at $\phi=\pi$. Similarly to the translational symmetric case, this degeneracy can also be explained by mutually anticommuting symmetry operations $U_{\mathcal{A}}(\pi) R$ and $F$ if there is no magnetic field and $\sum_{j \in \mathcal{A}} S_{j} \notin \mathbb{Z}$, where $R$ denotes the reflection. Similar arguments can be applied to the molecular magnets with Dzyaloshinsky-Moriya interactions. We can also apply this argument to the Majumdar-Ghosh (MG) model of length $2 n \quad(n \in \mathbb{N}, n \geq 3)$ with a periodic boundary condition. ${ }^{39}$ It gives a gap closing under the twist which is consistent with the doubly degenerate ground state at $\phi=0$ and $\pi$. In fact, as we show in Appendix D, the ground state of this model is always doubly degenerate for a certain twist with any $\phi$.

\section{CONCLUSION}

As for a quantum liquid with finite excitation energy gap, one may construct a quantum local order parameter by the Berry phase associated with a local gauge twist assuming the energy gap never closes even if the local twist exists. Generically speaking, this condition may not be always satisfied and it should be considered for each cases, since the energy gap can be collapsed by the local twist.

On the contrary, this simple observation brings an interesting and useful outcome. Since the local gauge twist is gauge dependent, a local gauge transformation of the Hamiltonian brings a change for the local twist. Of course, the local gauge transformation is just a unitary transformation and does not change the spectrum. The wave function gets modified but the change is tractable. Then as for some class of the Hamiltonians, the local gauge invariance of the Hamiltonian imposes a strong constraint for the local order parameters by the Berry phases. These Berry phases as the local order parameters reflect quantum responses for the local perturbation and respect a symmetry of the Hamiltonian. This also provides another constraint for the local order parameters. In some cases, these two constraints are contradicting. It implies that the Berry phases cannot be well defined, that is, the finite-energy gap of the Hamiltonian collapses by the local twists. For example, the Berry phase pattern has to be uniform for integer-spin Heisenberg chains and should be alternating for half-integer chains. It implies a gap closing for the translational invariant antiferromagnetic Heisenberg chains with the half-integer spin (in the finite system). As is well known, it is related to the excitation gap for an infinite system (LSM theorem).

The argument of the present paper is generic, however, we discussed some of translationally invariant spin ladders 
and spinless fermionic systems to be specific. Generalization for the non-Abelian case which includes a discussion for a (approximate) degeneracy, such as the topological degeneracy, is treated. The reflection symmetry is treated as well.

\section{ACKNOWLEDGMENTS}

H.K. was supported by JSPS (No. 1811271). The work of Y.H. was supported in part by Grants-in-Aid for Scientific Research No. 20340098 and No. 20654034 from JSPS, and No. 220029004 (Physics of New Quantum Phases in Super Clean Materials) and No. 20046002 (Novel States of Matter Induced by Frustration) on priority areas from MEXT.

\section{APPENDIX A: NON-ABELIAN BERRY PHASES}

Here let us review a Berry phase of a multiplet, which naturally includes a non-Abelian extension. Although this is given by one of the authors before, ${ }^{19-22}$ we give here a compact self-contained presentation for the readers' convenience.

Now let us take a parameter dependent Hamiltonian $H(x)$ of a finite dimensions $N, x \in V$ where $V$ is a parameter space. Then taking a closed curve $C$ in $V$ and assuming the lowest $M$ eigenvalues are separated from the other as

$$
\epsilon_{M}(x)<\epsilon_{M+1}(x), x \in C,
$$

where $\left\{\epsilon_{1}, \cdots \epsilon_{M}\right\}$ are a set of the $M$-lowest eigenvalues $\left(\epsilon_{j}\right.$ is the lowest $j$ th eigenvalue). With this generic gap opening condition, ${ }^{19-22}$ the following multiplet $\Psi(x)$ is well defined $(x \in C)$ :

$$
\begin{gathered}
\Psi(x)=\left(\left|\psi_{1}(x)\right\rangle, \cdots,\left|\psi_{M}(x)\right\rangle\right), \\
H(x)\left|\psi_{j}(x)\right\rangle=\epsilon_{j}(x)\left|\psi_{j}(x)\right\rangle .
\end{gathered}
$$

Assuming possible level crossings within this lowest $M$ states, we allow following $U(M)$ gauge transformation within the multiplet

$$
\Psi=\Psi^{\prime} \omega, \quad \omega \in U(M) .
$$

When the lowest $M$ states are $M$-fold degenerate, this $U(M)$ ambiguity is apparent and inevitable. Here we allow this to characterize the linear space spanned by the $M$ states. Considering this multiplet in modulo $U(M)$, the non-Abelian Berry connection (gauge potential one form) is defined as

$$
\begin{aligned}
A=A_{\mu} d x^{\mu} & =\Psi^{\dagger} d \Psi=\left[\begin{array}{ccc}
\left\langle\psi_{1} \mid d \psi_{1}\right\rangle & \cdots & \left\langle\psi_{1} \mid d \psi_{M}\right\rangle \\
\vdots & \ddots & \vdots \\
\left\langle\psi_{M} \mid d \psi_{1}\right\rangle & \cdots & \left\langle\psi_{M} \mid d \psi_{M}\right\rangle
\end{array}\right], \\
A_{\mu} & =\left[\begin{array}{ccc}
\left\langle\psi_{1} \mid \partial_{\mu} \psi_{1}\right\rangle & \cdots & \left\langle\psi_{1} \mid \partial_{\mu} \psi_{M}\right\rangle \\
\vdots & \ddots & \vdots \\
\left\langle\psi_{M} \mid \partial_{\mu} \psi_{1}\right\rangle & \cdots & \left\langle\psi_{M} \mid \partial_{\mu} \psi_{M}\right\rangle
\end{array}\right],
\end{aligned}
$$

where $\left\{x^{\mu}\right\}$ is a local coordinate of the parameter space $V$. Above $U(M)$ gauge transformation gives a change in the gauge potential as

$$
A=\omega^{-1} \Psi^{\prime \dagger}\left(d \Psi^{\prime} \omega+\Psi^{\prime} d \omega\right)=\omega^{-1} A^{\prime} \omega+\omega^{-1} d \omega,
$$

where $A^{\prime}=\left(\Psi^{\prime}\right)^{\dagger} d \Psi^{\prime}$. Here, we assume the multiplet is normalized as

$$
\Psi^{\dagger} \Psi=\left(\Psi^{\prime}\right)^{\dagger} \Psi^{\prime}=E_{M},
$$

where $E_{M}$ is a unit matrix of the dimension $M$.

Now let us define the Berry phase $\gamma_{A}(C)$ of this nonAbelian Berry connection (along the curve $C$ ) as

$$
i \gamma_{A}(C)=\int_{C} \operatorname{Tr} A=\int_{C}\left(\operatorname{Tr} A_{\mu}\right) d x^{\mu} .
$$

This is apparently gauge dependent and the change in the multiplet brings

$$
\begin{gathered}
\gamma_{A}=\gamma_{A^{\prime}}+\Delta, \\
\Delta=-i \int_{C} \operatorname{Tr} d \log \omega=\int_{C} d \operatorname{Arg} \operatorname{det} \omega .
\end{gathered}
$$

It implies that the Berry phase itself is gauge dependent but is well defined in modulo $2 \pi$ assuming the gauge transformation $\omega$ is well defined (as a single valued matrix) on the curve $C$, i.e.,

$$
\gamma_{A} \equiv \gamma_{A^{\prime}} \quad \bmod 2 \pi .
$$

This gauge dependence is clearly demonstrated when we fix the gauge. The gauge of the multiplet is explicitly fixed following the general procedure given by one of the present authors as

$$
\begin{gathered}
\Psi_{\Phi}=\Psi_{\Phi}^{U} N_{\Phi}^{-1 / 2}, \\
\Psi_{\Phi}^{U}=P \Phi,
\end{gathered}
$$

where $\Phi$ is an arbitrary fixed multiplet and $P=\Psi \Psi^{\dagger}$ is a projection into the multiplet space which is gauge independent $\left(P=P^{\prime}\right)$. The normalization matrix $N_{\Phi}$ is chosen as

$$
\Psi_{\Phi}^{\dagger} \Psi_{\Phi}=E_{M},
$$

which is realized by taking as

$$
\begin{gathered}
N_{\Phi}=\left(\Psi_{\Phi}^{U}\right)^{\dagger} \Psi_{\Phi}^{U}=\eta_{\Phi}^{\dagger} \eta_{\Phi}, \\
\eta_{\Phi}=\Psi^{\dagger} \Phi
\end{gathered}
$$

Note that $\eta_{\Phi}$ is gauge dependent but the normalization matrix $N_{\Phi}$ is a gauge invariant. As for the arbitrarily taken multiplet $\Phi$, the gauge of the multiplet and the Berry connection is fixed by this procedure assuming that $N_{\Phi}^{-1 / 2}$ exists, that is

$$
\operatorname{det}_{M} N_{\Phi}=\left|\operatorname{det}_{M} \Psi^{\dagger} \Phi\right|^{2} \neq 0 .
$$

The above condition has a codimension 2 and it is generically satisfied on the curve (one-dimensional manifold). Continuous deformation of the gauge fixing multiplet $\Phi$, keeping $\mid$ det $\Psi^{\dagger} \Phi \mid \neq 0$, gives a small gauge transformation. On the other hand, if two gauge fixing multiplets $\Phi_{1}, \Phi_{2}$ cannot be deformed unless the normalization determinant vanishes, they define a large gauge transformation. The gauge transformation between them is generically given as follows:

$$
\begin{gathered}
\Psi_{2}(x)=\Psi_{1}(x) \omega_{12}(x), \\
\omega_{12}(x)=N_{1}^{1 / 2} \eta_{1}^{-1} \eta_{2} N_{2}^{-1 / 2},
\end{gathered}
$$




$$
\begin{gathered}
\Psi_{i}=P \Phi_{i} N_{i}^{-1 / 2}, \\
N_{i}=\eta_{i}^{\dagger} \eta_{i}, \quad \eta_{i}=\Psi^{\dagger} \Phi_{i}, \quad i=1,2,
\end{gathered}
$$

for ${ }^{\forall}$, det $\eta_{i} \neq 0$. This condition is generically satisfied on the curve $C$. Its unitarity is confirmed as

$$
\begin{aligned}
\omega_{12}^{\dagger} \omega_{12} & =N_{2}^{-1 / 2} \eta_{2}^{\dagger}\left(\eta_{1}^{\dagger}\right)^{-1} N_{1}^{1 / 2} N_{1}^{1 / 2} \eta_{1}^{-1} \eta_{2} N_{2}^{-1 / 2} \\
& =N_{2}^{-1 / 2} \eta_{2}^{\dagger}\left(\eta_{1}^{\dagger}\right)^{-1} \eta_{1}^{\dagger} \eta_{1} \eta_{1}^{-1} \eta_{2} N_{2}^{-1 / 2} \\
& =N_{2}^{-1 / 2} \eta_{2}^{\dagger} \eta_{2} N_{2}^{-1 / 2}=E_{M} .
\end{aligned}
$$

\section{APPENDIX B: QUANTIZATION OF THE BERRY PHASES}

Now let us review a quantization of the non-Abelian Berry phase assuming the $M$-dimensional multiplet is invariant under the antiunitary operator $\Theta=K U$, where $U$ is a parameter independent unitary operator $(d U=0)$ and $K$ is a complex-conjugate operation

$$
\Psi^{\Theta}=\Theta \Psi
$$

From a simple observation

$$
A^{\Theta}=\left(\Psi^{\Theta}\right)^{\dagger} d \Psi^{\Theta}=\left(\Psi^{\dagger}\right)^{*}\left(U^{\dagger} U\right)^{*}(d \Psi)^{*}=\left(\Psi^{\dagger} d \Psi\right)^{*}=A^{*},
$$

we have

$$
\operatorname{Tr} A^{\Theta}=-\operatorname{Tr} A .
$$

Since $A$ is antihermitian as

$A^{\dagger}=\left(\Psi^{\dagger} d \Psi\right)^{\dagger}=d \Psi^{\dagger} \Psi=d\left(\Psi^{\dagger} \Psi\right)-\Psi^{\dagger} d \Psi=d E_{M}-A=-A$, it implies

$$
\operatorname{Tr} A^{\dagger}=\operatorname{Tr} A^{*}=-\operatorname{Tr} A .
$$

Then the corresponding Berry phase of the multiplet $\Psi^{\Theta}$ $=\Theta \Psi$ is given by

$$
\gamma_{A^{\Theta}}=-\gamma_{A} .
$$

The invariance of the multiplet, i.e., invariance of the multiplet space, implies a gauge equivalence $\left({ }^{\exists} \omega\right)$ :

$$
\Psi^{\Theta}=\Psi \omega .
$$

It implies

$$
\gamma_{A^{\Theta}} \equiv \gamma_{A} \quad \bmod 2 \pi .
$$

With the help of Eq. (B1), allowed values of $\gamma_{A}$ are restricted to

$$
\gamma_{A} \equiv 0, \pi \bmod 2 \pi .
$$

It is a quantization of the non-Abelian Berry phases protected by the antiunitary symmetry.

\section{APPENDIX C: NUMERICAL EVALUATION OF THE BERRY PHASES}

Here we present a numerically efficient way to evaluate the Berry phases of the non-Abelian Berry connection. ${ }^{19-22,40}$
Let us first parametrize the curve $C$ in the parameter space as $C=\{x(t) \mid t \in[0,1]\}, x(0)=x(1)$. Then discretizing the curve as

$$
x_{n}=x(n \Delta t), \quad \Delta t=1 / L,
$$

let us define a non-Abelian lattice Berry phase $\Gamma_{L}$ as

$$
\begin{aligned}
\Gamma_{L} & =-\operatorname{Arg} \operatorname{det}_{M}\left[\left(\Psi_{1}^{\dagger} \Psi_{2}\right) \cdots\left(\Psi_{L-1}^{\dagger} \Psi_{L}\right)\right] \\
& =-\operatorname{Im} \log \operatorname{det}_{M}\left[\left(\Psi_{1}^{\dagger} \Psi_{2}\right) \cdots\left(\Psi_{L-1}^{\dagger} \Psi_{L}\right)\right] \\
& =-\operatorname{Im} \operatorname{Tr}_{M} \log \left[\left(\Psi_{1}^{\dagger} \Psi_{2}\right) \cdots\left(\Psi_{L-1}^{\dagger} \Psi_{L}\right)\right],
\end{aligned}
$$

where $\Psi_{\ell}=\Psi\left(x_{\ell}\right)$. When the gauge is smoothly fixed and allowed to expand the multiplet, we have

$$
\Psi_{\ell}^{\dagger} \Psi_{\ell+1} \approx E_{M}+\Delta t \Psi_{\ell}^{\dagger} \partial_{t} \Psi_{\ell} .
$$

Therefore we also have

$$
\begin{aligned}
\Gamma_{L} & \approx \operatorname{Im} \operatorname{Tr}_{M} \sum_{\ell} \Delta t \Psi_{\ell}^{\dagger} \partial_{t} \Psi_{\ell} \rightarrow \operatorname{Im} \operatorname{Tr} \int_{C} \Psi^{\dagger} d \Psi \\
& =-i \int_{C} \operatorname{Tr} \Psi^{\dagger} d \Psi
\end{aligned}
$$

in the limit $L \rightarrow \infty$. It implies this $\Gamma_{L}$ is a lattice analog of the Berry phase $\gamma_{A}$. Further note that this $\Gamma_{L}$ is apparently gauge invariant under the gauge transformation

$$
\Psi_{\ell}=\Psi_{\ell}^{\prime} \omega_{\ell} .
$$

It implies the smooth gauge fixing which we assume is not necessary at all. The continuum correspondent $\gamma_{A}$ is gauge dependent but this lattice analog is not.

When the multiplet is invariant under the antiunitary operation, $\Gamma_{L}$ gets modified as

$$
\begin{aligned}
\Gamma_{L}^{\Theta} & \equiv-\operatorname{Arg} \operatorname{det}_{M}\left[\left(\Psi_{1}^{\Theta \dagger} \Psi_{2}^{\Theta}\right) \cdots\left(\Psi_{L-1}^{\Theta \dagger} \Psi_{L}^{\Theta}\right)\right] \\
& =-\operatorname{Arg} \operatorname{det}_{M}\left[\left(\Psi_{1}^{\dagger} \Psi_{2}\right)^{*} \cdots\left(\Psi_{L-1}^{\dagger} \Psi_{L}\right)^{*}\right] \\
& =+\operatorname{Arg} \operatorname{det}_{M}\left[\left(\Psi_{1}^{\dagger} \Psi_{2}\right) \cdots\left(\Psi_{L-1}^{\dagger} \Psi_{L}\right)\right] \\
& =-\Gamma_{L},
\end{aligned}
$$

and it implies

$$
\Gamma_{L}=0, \pi .
$$

This lattice analog is also quantized even in the finite discretization. Now the reason of the numerical efficiency should be clear.

\section{APPENDIX D: EXACT GROUND STATES OF THE TWISTED MAJUMDAR-GHOSH MODEL}

In this appendix, we explicitly confirm that the ground state of the twisted MG model is always doubly degenerate for some pattern of twists with any $\phi$. The Hamiltonian of the twisted MG model is generally given by the following form: 


$$
H(\{\phi\})=\frac{3 J}{4} \sum_{j=1}^{L} P_{j-1, j, j+1}\left(\phi_{j-1, j}, \phi_{j-1, j+1}, \phi_{j, j+1}\right),
$$

where $L$ denotes the total number of sites and $\phi_{i, j}$ is the twist introduced on the link $(i, j)$. This model is a spin-1/2 model. The operator $P_{i j k}$ is defined for a triangle consisting of the sites $i, j$, and $k$, and is written as

$$
P_{i j k}\left(\phi_{i j}, \phi_{i k}, \phi_{j k}\right)=\frac{2}{3}\left[h_{i j}\left(\phi_{i j}\right)+h_{i k}\left(\phi_{i k}\right)+h_{j k}\left(\phi_{j k}\right)\right],
$$

with

$$
h_{i j}\left(\phi_{i j}\right)=\frac{1}{2}\left(e^{i \phi_{i j}} S_{i}^{+} S_{j}^{-}+e^{-i \phi_{i j}} S_{i}^{-} S_{j}^{+}\right)+S_{i}^{z} S_{j}^{z}+\frac{1}{4} .
$$

Here we note that $P_{i j k}(0,0,0)$ is a projection operator which projects the total spin for the triangle $\boldsymbol{S}_{t}=\boldsymbol{S}_{1}+\boldsymbol{S}_{2}+\boldsymbol{S}_{3}$ onto the subspace of magnitude $3 / 2$. The untwisted Hamiltonian $H(\{0\})$ reproduces the original MG model $^{39}$ as

$$
H(\{0\})=J \sum_{j=1}^{L}\left(S_{j} \cdot S_{j+1}+\frac{1}{2} S_{j} \cdot S_{j+2}+\frac{3}{8}\right) .
$$

We shall consider the model with a periodic boundary condition and impose the following condition: $\boldsymbol{S}_{L+j}=\boldsymbol{S}_{j}$. In this case, the ground state of $H(\{0\})$ is well-known singlet product state and is doubly degenerate. The Hamiltonian Eq. (D1) is the sum of local Hamiltonians which of each acts on a single triangle. Therefore, if one finds a state minimizing any $P_{i j k}$ in Eq. (D1), then it is a ground state.

Let us now construct ground states of the model for a specific pattern of twists. Figure 3(a) shows this pattern where $\phi_{l . l+1}=\phi_{l, l+2}=\phi_{l+1, l+2}=\phi$, otherwise zero. In this case, the reflection symmetric line is taken to be through the site $l+2$. By an explicit calculation or using the fact that $P_{i j k}(0, \phi, \phi)=U_{k}(\phi) P_{i j k} U_{k}^{\dagger}(\phi) \quad$ with $\quad U_{k}(\phi)=\exp [i(1 / 2$ $\left.\left.-S_{k}^{z}\right) \phi\right]$, one can confirm that the states schematically shown in Figs. 3(b) and 3(c) are the ground states which minimize any local Hamiltonian. They are zero-energy ground states and explicitly given by
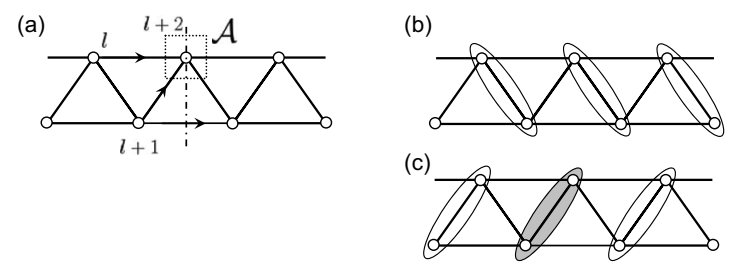

FIG. 3. (a) A pattern of twists for the Majumdar-Ghosh model. The arrows indicate twists on links while dotted square denotes $\mathcal{A}$ where the local unitary transformation $U_{l+2}(\phi)$ is performed. The system is reflection symmetric about the dashed line. (b) and (c) represent two exact ground states of the twisted MG model. They are called $\left|G_{1}\right\rangle$ and $\left|G_{2}\right\rangle$ in the text. Open and shaded ovals correspond to the singlet state and the twisted one Eq. (D5), respectively.

$$
\begin{gathered}
\left|G_{1}\right\rangle=\cdots[l, l+1][l+2, l+3][l+4, l+5] \cdots, \\
\left|G_{2}\right\rangle=\cdots[l-1, l]\{l+1, l+2\}(\phi)[l+3, l+4] \cdots,
\end{gathered}
$$

where $[i, j]=\left(|\uparrow \downarrow\rangle_{i j}-|\downarrow \uparrow\rangle_{i j}\right) / \sqrt{2}$ is the singlet state while $\{i, j\}(\phi)$ is the twisted singlet state and is defined as

$$
\{i, j\}(\phi)=\frac{1}{\sqrt{2}}\left(e^{i \phi_{i j} / 2}|\uparrow \downarrow\rangle_{i j}-e^{-i \phi_{i j} / 2}|\downarrow \uparrow\rangle_{i j}\right) .
$$

We should note here that the unitary equivalence of the local Hamiltonian does not mean the equivalence of the twisted and the original Hamiltonians. Actually, there are loops where we cannot eliminate the flux through them by any unitary transformations. We have also numerically confirmed that the whole energy spectrum depends on $\phi$ for $L=4,6$. It means that the twisted model cannot be obtained from the untwisted one by any unitary transformation. In the figure, an open oval represents the singlet state, while the shaded one is a twisted singlet. The first ground state $\left|G_{1}\right\rangle[\mathrm{Fig} .3(\mathrm{~b})]$ is also a ground state of $H(\{0\})$. In other words, one of the ground states of the MG model is stable under the twist. On the other hand, the second one $\left|G_{2}\right\rangle$ [Fig. 3(c)] is obtained from the other ground state of $H(\{0\})$ by a local unitary transformation $U_{k}(\phi)$. It is important to note again that while the ground-state energy is always zero during the twist, there is no unitary transformation connecting $H(\{\phi\})$ and $H(\{0\})$ for the twist shown in Fig. 3(a).

\footnotetext{
*hirano@pothos.t.u-tokyo.ac.jp

${ }^{\dagger}$ katsura@appi.t.u-tokyo.ac.jp

${ }^{\ddagger}$ Corresponding author. Present address: Institute of Physics, University of Tsukuba: hatsugai@sakura.cc.tsukuba.ac.jp

${ }^{1}$ E. H. Lieb, T. Schultz, and D. J. Mattis, Ann. Phys. (N.Y.) 16, 407 (1961).

${ }^{2}$ I. Affleck and E. H. Lieb, Lett. Math. Phys. 12, 57 (1986).

${ }^{3}$ I. Affleck, Phys. Rev. B 37, 5186 (1988).

${ }^{4}$ M. Oshikawa, Phys. Rev. Lett. 84, 1535 (2000).

${ }^{5}$ M. Yamanaka, M. Oshikawa, and I. Affleck, Phys. Rev. Lett. 79, 1110 (1997)

${ }^{6}$ M. B. Hastings, Phys. Rev. B 69, 104431 (2004).
}

${ }^{7}$ G. Misguich, C. Lhuillier, M. Mambrini, and P. Sindzingre, Eur. Phys. J. B 26, 167 (2002).

${ }^{8}$ R. B. Laughlin, Phys. Rev. B 23, 5632 (1981).

${ }^{9}$ B. I. Halperin, Phys. Rev. B 25, 2185 (1982).

${ }^{10}$ Y. Hatsugai, Phys. Rev. B 48, 11851 (1993).

${ }^{11}$ Y. Hatsugai, Phys. Rev. Lett. 71, 3697 (1993).

${ }^{12}$ F. D. M. Haldane, Phys. Lett. 93A, 464 (1983).

${ }^{13}$ T. Kennedy, J. Phys.: Condens. Matter 2, 5737 (1990).

${ }^{14}$ M. Hagiwara, K. Katsumata, I. Affleck, B. I. Halperin, and J. P. Renard, Phys. Rev. Lett. 65, 3181 (1990).

${ }^{15}$ J. P. Renard, M. Verdaguer, L. P. Regnault, W. A. C. Erkelens, J. Rossat-Mignod, and W. G. Stirling, Europhys. Lett. 3, 945 
(1987).

${ }^{16}$ K. Katsumata, H. Hori, T. Takeuchi, M. Date, A. Yamagishi, and J. P. Renard, Phys. Rev. Lett. 63, 86 (1989).

${ }^{17}$ X. G. Wen, Phys. Rev. B 40, 7387 (1989).

${ }^{18}$ Y. S. Wu, Y. Hatsugai, and M. Kohmoto, Phys. Rev. Lett. 66, 659 (1991).

${ }^{19}$ Y. Hatsugai, J. Phys. Soc. Jpn. 73, 2604 (2004).

${ }^{20}$ Y. Hatsugai, J. Phys. Soc. Jpn. 74, 1374 (2005).

${ }^{21}$ Y. Hatsugai, J. Phys. Soc. Jpn. 75, 123601 (2006).

${ }^{22}$ Y. Hatsugai, arXiv:cond-mat/0603230 (unpublished).

${ }^{23}$ I. Maruyama and Y. Hatsugai, J. Phys. Soc. Jpn. 76, 113601 (2007).

${ }^{24}$ T. Hirano, H. Katsura, and Y. Hatsugai, Phys. Rev. B 77, 094431 (2008).

${ }^{25}$ M. V. Berry, Proc. R. Soc. London A392, 45 (1984).

${ }^{26}$ M. Kolb, Phys. Rev. B 31, 7494 (1985).

${ }^{27}$ V. E. Korepin and A. C. T. Wu, Int. J. Mod. Phys. B 5, 497 (1991).

${ }^{28}$ Y. Hatsugai, J. Phys.: Condens. Matter 19, 145209 (2007).

${ }^{29}$ M. Oshikawa, M. Yamanaka, and I. Affleck, Phys. Rev. Lett. 78,
1984 (1997).

${ }^{30}$ Y. Hatsugai, Phys. Rev. B 56, 12183 (1997).

${ }^{31}$ E. Dagotto and T. M. Rice, Science 271, 618 (1996).

${ }^{32}$ S. Dell' Aringa, E. Ercolessi, G. Morandi, P. Pieri, and M. Roncaglia, Phys. Rev. Lett. 78, 2457 (1997).

${ }^{33}$ K. Kawano and M. Takahashi, J. Phys. Soc. Jpn. 66, 4001 (1997).

${ }^{34}$ T. Fukui and N. Kawakami, Phys. Rev. B 55, R14709 (1997).

${ }^{35}$ H. Tsunetsugu, Y. Hatsugai, K. Ueda, and M. Sigrist, Phys. Rev. B 46, 3175 (1992).

${ }^{36}$ Y. Hatsugai, M. Kohmoto, and Y. S. Wu, Phys. Rev. B 43, 10761 (1991).

${ }^{37}$ Y. S. Wu, Y. Hatsugai, and M. Kohmoto, Phys. Rev. Lett. 66, 659 (1991).

${ }^{38}$ Q. Niu, D. J. Thouless, and Y. S. Wu, Phys. Rev. B 31, 3372 (1985).

${ }^{39}$ C. K. Majumdar and D. K. Ghosh, J. Math. Phys. 10, 1388 (1969).

${ }^{40}$ T. Fukui, Y. Hatsugai, and H. Suzuki, J. Phys. Soc. Jpn. 74, 1674 (2005). 